\title{
Content Delivery in Vehicular Ad Hoc Networks
}

\author{
Fabrício A. Silva ${ }^{1}$, \\ Linnyer B. Ruiz ${ }^{2}$ (Advisor), Antonio A. F. Loureiro ${ }^{1}$ (Co-Advisor) \\ ${ }^{1}$ Departamento de Ciência da Computação (UFMG) \\ ${ }^{2}$ Departamento de Informática (UEM)
}

\begin{abstract}
Vehicular Ad Hoc Network (VANET) applications are emerging to reality with the objective of making traffic safer, less congested, more informative, and enjoyable. With the advance of such applications, comes the need for content delivery solutions, since the efficient delivery of content is a fundamental requirement for most vehicular network applications. The main objective of this thesis is to investigate how existing concepts can be adapted and applied to VANETs and to propose content delivery solutions for this kind of network.
\end{abstract}

\section{Introduction}

Vehicular Ad Hoc Networks (VANETs) are migrating from theory to practice mainly due to the great interest of vehicle's manufacturers to provide new on-road services to their clients [Zeadally et al. 2012]. This kind of network consists of vehicles with on-board wireless communication facilities that are able to establish ad hoc communication with their peers as well as with infrastructure stations [Wang and Li 2009]. VANET applications are emerging to reality with the objective of making traffic safer, less congested, more informative, and enjoyable [Lee et al. 2014, Costa-Montenegro et al. 2012]. With the advance of such applications, comes the need for content delivery solutions, since the efficient delivery of content is a fundamental requirement for most vehicular network applications, such as information, advertisement, and entertainment systems. Nevertheless, the VANET particular characteristics make the task of delivering content easier said than done [Gerla et al. 2014].

Usually, two approaches have been used to deliver content in the traditional Internet [Passarella 2012]: Content Delivery Networks (CDN) and Peer-to-Peer (P2P). Several characteristics of VANETs and their applications suggest that pure CDN and P2P models, as originally conceived for the Internet, are not suitable for them. First, many applications are referred to as push-based, meaning that content should be pushed to the clients even in the absence of a request, like an accident notification, for example. In addition, contacts in VANETs are intermittent, making the establishment and maintenance of endto-end links very difficult. Furthermore, the deployment of surrogate servers in a largescale urban scenario is a costly, time-consuming task. When it comes to content, several entities are potential sources, such as vehicles, Intelligent Transportation System (ITS) solutions, Wireless Sensor Networks (WSNs), mobile users, among others. Moreover, content sources and clients move in considerable speed, causing constant changes in the network topology. Finally, content in many applications is location- and time-dependent, meaning it is valid only inside a region of interest (RoI) during a given period.

\subsection{Objective and Contributions}

Given the need for more efficient vehicular content delivery solutions, the hypothesis of the thesis is defined as: the adoption of a hybrid model that inherits and adapts concepts 
from both CDN and P2P will improve the performance of VANET applications in terms of content availability, quality of experience, and delivery cost. Therefore, the main objective is to investigate how CDN and P2P concepts can be adapted and applied to VANETs, forming a Vehicular Content Delivery Network (VCDN), and propose solutions that validate the aforementioned hypothesis.

The main contributions of the thesis are summarized as follows:

1. A novel content delivery model, called VCDN, that inherits and adapts concepts from CDN and P2P, and a framework to help application designers to define the most appropriate architecture and techniques to be adopted by their applications;

2. Two innovative solutions based on our proposed VCDN model developed to make content available in city-wide and region-wide scenarios. These solutions, in addition to validate our hypothesis, advance the state-of-the-art since they are based on novel methods that consider origin-destination points as input;

3. Characterization results that lead to important insights on how to select appropriate replica vehicles in content delivery applications;

4. A survey of VANET content delivery solutions found in the literature organized according to their techniques and architectures.

\section{Background}

In Chapter 2 of the thesis, we present an in-depth survey of the literature in terms of content delivery concepts applied to VANETs. Basically, there are two major challenges when designing content delivery solutions [Peng 2004]. The first one is the issue of selecting good places to replicate content. The second challenge is related to the discovery of the most appropriate server, either origin or surrogate, and the content delivery itself. These issues are the two fundamental building blocks that compose a content delivery system, and are even more challenging in such dynamic environments as VANETs.

In the thesis, we classify the studies from the literature under their solution basis and architecture perspectives, as shown in Figure 1. This detailed survey guided us to a promising challenge in the content delivery for vehicular networks, which is the proposing of a novel model that considers the VANET characteristics.

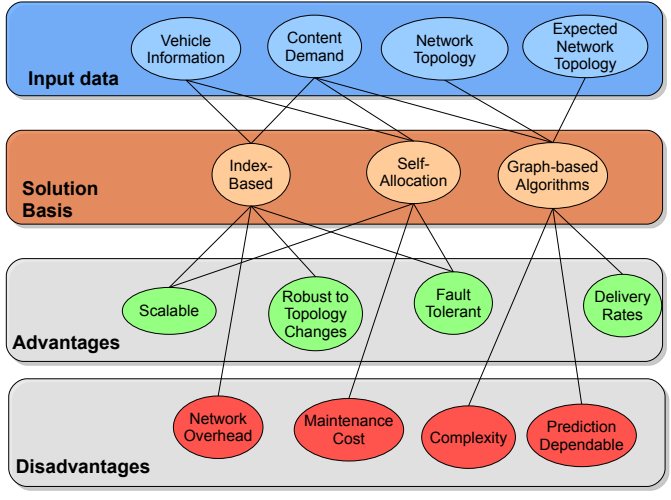

(a) Replica Allocation Solutions

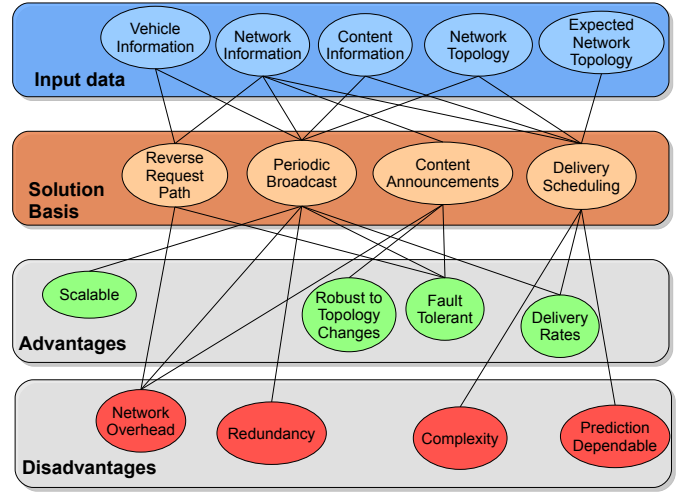

(b) Content Delivery Solutions

Figure 1. Replica allocation and content delivery solutions classification.

This is the first contribution of the thesis reported in the following publications: 
1. [Journal A1, Impact Factor: 3.37] Fabrício A. Silva; Azzedine Boukerche; Thais R. M. Braga Silva; Linnyer B. Ruiz; Eduardo Cerqueira; Antonio A. F. Loureiro, "Vehicular Networks: A New Challenge for Content Delivery-based Applications". ACM Computing Surveys (To Appear)

2. [Conference] Fabrício A. Silva; Azzedine Boukerche; Thais R. M. B. Silva; Linnyer B. Ruiz; Eduardo Cerqueira; Antonio A. F. Loureiro, "Content Replication and Delivery in Vehicular Networks". 4th ACM Symposium on Design and Analysis of Intelligent Vehicular Networks and Applications, (2014):127-132.

\section{Vehicular Content Delivery Model}

As observed in the results of the survey of the state-of-the-art, there is no model to help designers when developing VANET applications with content delivery demand. Therefore, in Chapter 3 of the thesis we propose a novel model called Vehicular Content Delivery Network (VCDN), where aspects of CDN and P2P are integrated, adapted, and extended to VANETs.

The original Content Delivery Network (CDN) and Peer-to-Peer (P2P) solutions have advantages and disadvantages. The main advantage of the pure CDN approach, considering the VANET context, is the infrastructured network comprised of the surrogate servers, leading to highly content availability. However, there is a high cost, in terms of money and time, to deploy and maintain the surrogate servers considering the large-scale scenario of VANETs. Furthermore, this approach is not fault-tolerant since the failure of a surrogate server may cause disconnections and, consequently, uncovered regions.

On the other hand, the main advantage of such pure P2P approach is its faulttolerance, since the failure of a provider is not perceived given the existence of other servers nearby. Nevertheless, there is a high cost in searching for content in terms of delay and network overhead, given the highly dynamic topologies posed by VANETs and the poor content availability that may occur.

Our proposed model takes advantage of the benefits from both CDN and P2P approaches, and extends some of their concepts considering the VANET's characteristics. On one hand, we exploit the idea of replicating content on surrogate servers running on Roadside Units (RSUs) to increase content availability. Furthermore, we extend such concept by allowing content to be replicated also in moving vehicles as well, which increases content availability and fault-tolerance. On the other hand, P2P concepts for distributed content discovery and delivery are exploited, together with incentive mechanisms that benefit cooperative peers. To reduce the overhead and time to find a content in the network, our model takes advantage of the infrastructured stations to track the content availability nearby and, then, to indicate potential providers to clients. To help the content replication and delivery tasks, our model includes a mobility management service that monitors mobility patterns and supports the decision of content replication, discovery, and delivery.

Figure 2 presents the VCDN system architecture and components. The VCDN components encompass all aspects of the overall content delivery problem for VANETs. In summary, our VCDN model is scalable, fault-tolerant, mobility-aware, and works even in the absence of infrastructure stations.

This is the second contribution of the thesis reported in the following publications: 


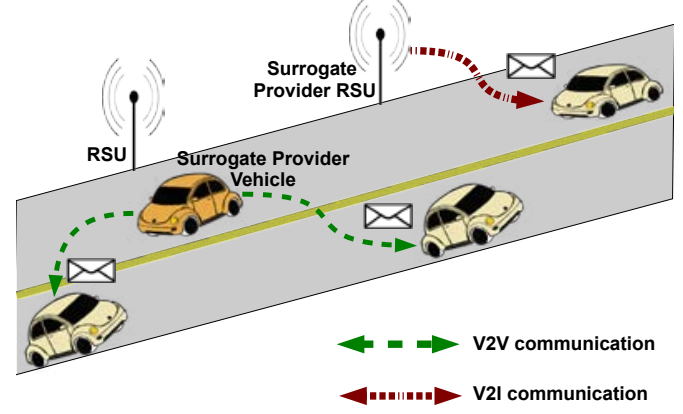

(a) VCDN System Architecture

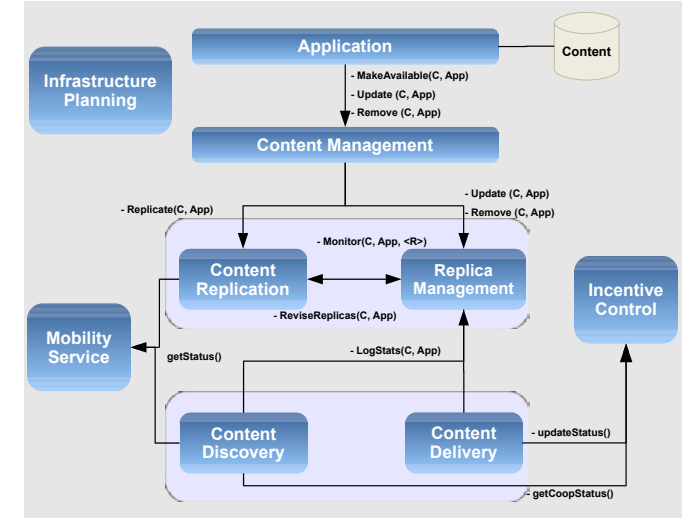

(b) VCDN Components

Figure 2. VCDN System Architecture and Components.

3. [Journal A1, Impact Factor: 5.41] Fabrício A. Silva; A. Boukerche; Thais R. M. B. Silva; Linnyer B. Ruiz; E. Cerqueira; Antonio A. F. Loureiro, "Vehicular Content Delivery Networks". IEEE Wireless Communications Magazine (To Appear)

4. [Conference B2] Fabrício A. Silva; Thais R. M. Braga Silva; Linnyer B. Ruiz; Eduardo Cerqueira; Antonio A. F. Loureiro, "Um modelo híbrido para entrega de conteúdo em redes veiculares". Simpósio Brasileiro de Redes de Computadores

\section{Evaluation}

We adopt the proposed model in real VANET applications with the objective of validating its benefits. However, before that, we present vehicular mobility characterization results to give insights on how to implement the VCDN building blocks.

In Chapter 4 of the thesis, we characterize a realistic, large-scale vehicular mobility trace under two perspectives: graph-based and origin-destination-based. In summary, the results reveal that some vehicles are more likely to be better replicas than others. Moreover, it was possible to infer a model to represent macroscopic aspects related to origin-destination (O-D) points. Given the difficulty to obtain a complete contact graph of a VANET, we argue that O-D points, which are easily obtained from navigation systems, can be useful for helping the design of content delivery solutions.

Therefore, in Chapter 5 of the thesis we implement the proposed VCDN model for two different applications: city-wide and region-wide. In the first, the content should be delivered to all vehicles in the network. In the second, on the other hand, the content should be delivered only to vehicles that are inside a region of interest during the content lifetime. Both solutions rely on the characterization results obtained in terms of O-D points.

We compare our solutions with others from the literature under important metrics to content delivery applications, such as coverage, the time a vehicle has to wait before being covered, redundancy, number of messages, among others. Figure 3 illustrates some of the results for the region-wide scenario. In summary, our solution (GO-DCR) leads to a higher coverage and a lower time to be covered (i.e., vehicles receive content earlier than in the baseline solutions). In addition, our solution leads to lower communication cost since it requires less vehicle-to-infrastructure (V2I) messages. 


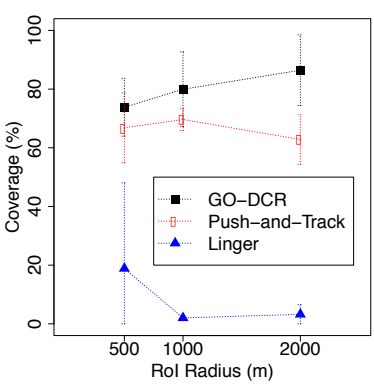

(a) Coverage

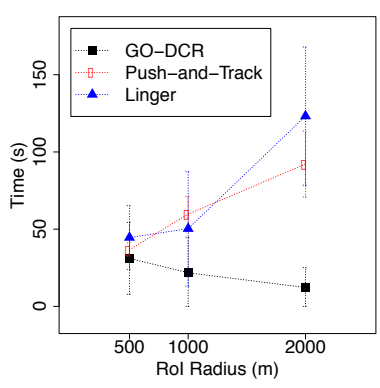

(b) Time to be Covered

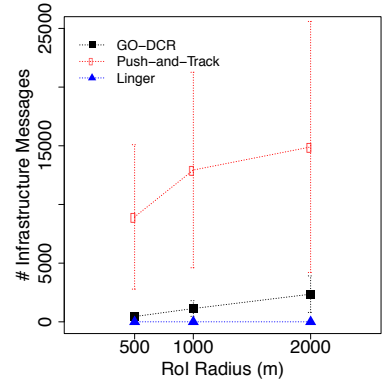

(c) Cost - V2I Messages

Figure 3. Sample results of the region-wide scenario.

In general, for both scenarios, our VCDN model was able to improve the content availability while reducing the communication cost. In addition to validate our proposed VCDN model, the contribution of this chapter also lies in the advance in the state-of-theart in concerns with content replication and delivery solutions for VANET applications.

The results of this part of the thesis were reported in the following publications:

5. [Journal B1, Impact Factor: 1.94] Fabrício A. Silva; Azzedine Boukerche; Thais R. M. B. Silva; Linnyer B. Ruiz; Antonio A. F. Loureiro, "Geo-localized Content Availability in VANETs". Ad Hoc Networks (Elsevier), 36(2):425-434 , Jan. 2016.

6. [Journal A1, Impact Factor: 2.64] Fabrício A. Silva; Azzedine Boukerche; Thais R. M. B. Silva; Fabrício Benevenuto; Linnyer B. Ruiz; Antonio A. F. Loureiro, "ODCRep: Origin-Destination-based Content Replication for Vehicular Networks". IEEE Transactions on Vehicular Technology 64(12):1-12, Dec. 2015.

7. [Journal A2, Impact Factor: 1.28] Fabrício A. Silva; A. Boukerche; Thais R. M. B. Silva; Linnyer B. Ruiz; Antonio A. F. Loureiro, "A novel macroscopic mobility model for vehicular networks". Computer Networks, 79(C):188-202, March 2015.

8. [Conference B1] Fabrício A. Silva; Clayson Celes; Azzedine Boukerche; Linnyer B. Ruiz; Antonio A. F. Loureiro, "Filling the Gaps of Vehicular Mobility Traces". ACM International Conference on Modeling, Analysis and Simulation of Wireless and Mobile Systems (MSWiM),(2015):47-54.

9. [Conference A2] Fabrício A. Silva; Azzedine Boukerche; Thais R. M. B. Silva; Linnyer B. Ruiz; Antonio A. F. Loureiro, "Geo-localized Content Replication for Vehicular Ad-hoc Networks". IEEE Symposium on Computers and Communications (ISCC),(2015):695-700.

10. [Conference A2] Fabrício A. Silva; Linnyer B. Ruiz; Antonio A. F. Loureiro, "Content Replication in Mobile Vehicular Ad-hoc Networks". IEEE Mobile Data Management (MDM),(2015):26-29.

11. [Conference A2] Fabrício A. Silva; Thais R. M. B. Silva; Fabrício Benevenuto; Linnyer B. Ruiz; Antonio A. F. Loureiro, "Improving information dissemination in vehicular networks by selecting appropriate disseminators". 28th IEEE International Conference on Advanced Information Networking and Applications (AINA),(2014):681-688.

12. [Conference A1] Fabrício A. Silva; Thais R. M. B. Silva; Linnyer B. Ruiz; Antonio A. F. Loureiro; Rafael Vicente, "On the improvement of vehicular macro- 
scopic mobility models". IEEE 78th Vehicular Technology Conference (VTCFall), (2013):1-5.

13. [Conference A1] Fabrício A. Silva; Thais R. M. B. Silva; Linnyer B. Ruiz; Antonio A. F. Loureiro, "ConProVA: A Smart Context Provisioning Middleware for VANET Applications". IEEE 77th Vehicular Technology Conference (VTCSpring), (2013):1-5.

14. [Conference B2] Fabrício A. Silva; Thais M. B. Silva; Linnyer B. Ruiz; Antonio A. F. Loureiro, "Um Middleware para provisionamento de contextos para redes veiculares". Simpósio Brasileiro de Redes de Computadores, (2013):615-628.

\section{Final Remarks and Impact}

In the thesis, we explored the field of content delivery for vehicular ad hoc networks (VANETs), with the hypothesis that concepts inherited from Content Delivery Network $(\mathrm{CDN})$ and Peer-to-Peer (P2P) and adapted to VANET applications would improve their performance. Given that, the objective was to investigate how those concepts could be applied to VANETs, and propose solutions that validate this hypothesis.

The impact of the thesis can be observed by the relevant results reported in very qualified journal and conference publications, as presented in the previous sections. All such publications are expected to contribute significantly with other researchers in this field. We highlight the work to appear in the ACM Computing Surveys (Impact Factor 3.37), which demonstrates our familiarity with the state-of-the-art in the area. We also state the importance of the other three journal papers published in the Ad Hoc Networks (Impact Factor 1.94), IEEE Transactions on Vehicular Technology (Impact Factor 2.64) and Computer Networks (Impact Factor 1.28), which reinforce that our work has been well accepted by the research community. Finally, we should mention the last journal paper that describes the overall VCDN model to appear in the IEEE Wireless Communications Magazine (Impact Factor: 5.41).

\section{References}

Costa-Montenegro, E., Quiñoy-García, F., González-Castaño, F., and Gil-Castineira, F. (2012). Vehicular entertainment systems: Mobile application enhancement in networked infrastructures. IEEE Vehicular Technology Magazine, 7(3):73-79.

Gerla, M., Wu, C., Pau, G., and Zhu, X. (2014). Content distribution in VANETs. Vehicular Communications, 1(1):3-12.

Lee, E., Lee, E.-k., and Gerla, M. (2014). Vehicular Cloud Networking: Architecture and Design Principles. IEEE Communications Magazine, (2):148-155.

Passarella, A. (2012). A survey on content-centric technologies for the current internet: Cdn and p2p solutions. Computer Communications, 35(1):1-32.

Peng, G. (2004). CDN: Content Distribution Network. arXiv preprint cs/0411069.

Wang, Y. and Li, F. (2009). Vehicular ad hoc networks. In Guide to wireless ad hoc networks, pages 503-525. Springer.

Zeadally, S., Hunt, R., Chen, Y.-S., Irwin, A., and Hassan, A. (2012). Vehicular ad hoc networks (VANETS): status, results, and challenges. Telecommunication Systems, 50(4):217-241. 